\title{
FORMACIÓN TÉCNICA DE NIVEL SUPERIOR Principales ejes de política, instrumentos y líneas de acción
}

Pilar Alamos*

* Jefa del Departamento de Centros de Formación Técnica del Ministerio de Educación 
El artículo se refiere a los ejes orientadores de políticas en la formación técnica de nivel superior.

This paper refers to the main policy guidelines to be followed in higher education of a technical nature. 


\section{FORMACIÓN TÉCNICA DE NIVEL SUPERIOR Principales ejes de política, instrumentos y líneas de acción}

La educación superior chilena ha logrado en los últimos años una mayor adaptación a los requerimientos actuales, gracias, entre otros factores, a la creación y desarrollo de los centros de formación técnica y los institutos profesionales; los programas técnicos; el reconocimiento de experiencia laboral o estudios previos; la educación a distancia y algunos esquemas de modularización de programas, transferencia interinstitucional y continuidad de estudios de un nivel de formación al siguiente.

Sin embargo estas experiencias siguen siendo excepciones para la forma en que opera nuestra educación superior, centrada en la universidad en detrimento de las instituciones no universitarias, orientada a responder a la demanda por carreras profesionales más que a los requerimientos del desarrollo productivo, en perjuicio del desarrollo del sector técnico, y caracterizada por planes de formación rígidos, cerrados a transferencias de otras carreras y otras instituciones.

En Chile, la oferta de carreras técnicas se caracteriza por una gran heterogeneidad y, a pesar de que la tendencia a la baja en el total de la matrícula se ha estabilizado a partir del año 2000, demostrando que las acciones emprendidas han tenido un efecto positivo, la cobertura de la formación técnica superior es mucho menor que la que el país requiere en esta etapa de su desarrollo económico. ${ }^{1}$

1. La apertura de carreras percibidas como de mejor calidad, la existencia de oportunidades de articulación en algunas instituciones y el otorgamiento de becas han empezado a revertir el proceso descendente de la matrícula. 
En este ámbito, la promoción de la calidad y el fomento de la equidad -principales objetivos de política de educación superior estrechamente ligados entre sí- encuentran una expresión particular. Así, por una parte se plantea mejorar la oferta existente y ampliar las alternativas de articulación horizontal y vertical, lo cual incide directamente en el atractivo de las opciones de formación técnica y el aumento de la demanda. Por otra, el establecimiento de ayudas y los mecanismos de apoyo contribuirán a que los potenciales estudiantes de bajos ingresos puedan proseguir estudios en este nivel.
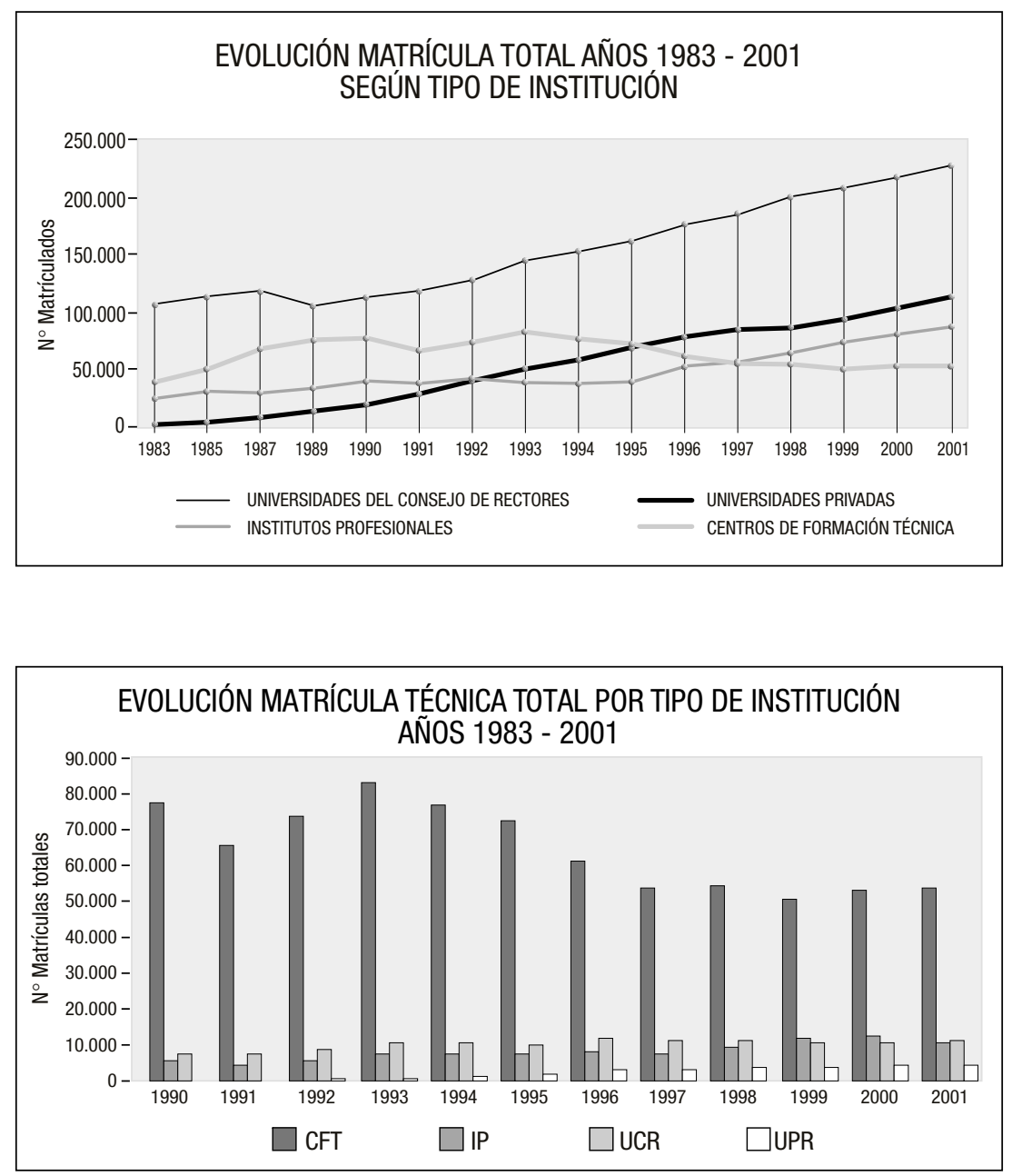


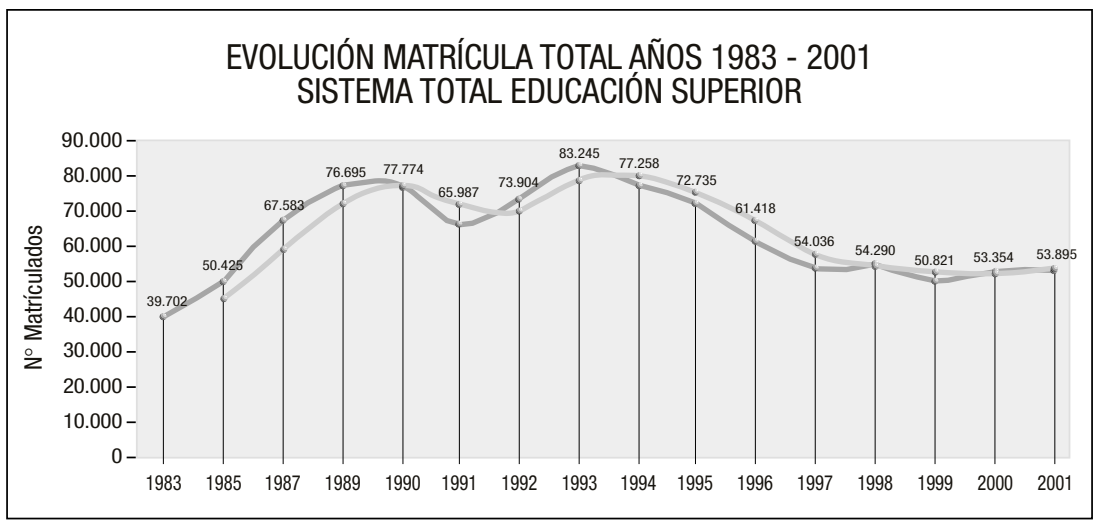

\section{PROMOCIÓN DE LA CALIDAD}

Una responsabilidad fundamental del Estado es fomentar la pertinencia y la calidad de la educación superior, elementos esenciales para la validez social de los programas y títulos que se ofrecen, así como para garantizar la fe pública involucrada en ellos.

En este contexto se precisa establecer con claridad el alcance y el significado del título de técnico de nivel superior, para llegar a una visión compartida sobre este tipo de recurso humano, basada en las exigencias reales del desarrollo del país.

La calidad depende no sólo de la definición de los objetivos de formación, sino también de la forma en que cada institución se organiza para lograrlos. En este sentido, por una parte, deben considerarse los aspectos curriculares y metodológicos, y por otra, los recursos disponibles para desarrollarlos de manera eficaz.

Igualmente, para lograr una educación técnica más pertinente y de calidad, es necesario definir perfiles profesionales, desarrollar enfoques curriculares orientados al logro de competencias de acción, así como aplicar modalidades educativas como "enseñanza dual" o "formación por alternancia". 
La integración del sector productivo en el proceso formativo es fundamental, ya que contribuye a dar claridad y coherencia a los itinerarios de formación en función de las demandas del mercado laboral; a incorporar la visión empresarial y la cultura de la empresa en los contenidos y nuevos métodos pedagógicos y a dar apoyo y credibilidad a esta estructura educativa en la comunidad y en el entorno profesional.

Otro tema que cabe destacar es la calidad del cuerpo docente que se desempeña en este segmento educacional. En la educación técnica de nivel superior el docente debe ser un tecnólogo de alto nivel, un profesional especializado en este particular tipo de enseñanza, comprometido en todos los ámbitos de la formación y, en general, capaz de organizar la formación continua con calidad.

Por último, en un contexto en que el financiamiento proviene casi exclusivamente de los aranceles pagados por los estudiantes, indirectamente el tamaño de las instituciones se convierte en un elemento central asociado a la calidad. En nuestro sistema, el 60\% de los centros de formación técnica tiene menos de 250 alumnos, y sólo el 18,5\% sobrepasa los 450 . La calidad, entonces, puede promoverse mediante incentivos para establecer fusiones, consorcios, asociaciones y otras estrategias que permitan desarrollar economías de escala y optimizar el uso de los recursos.

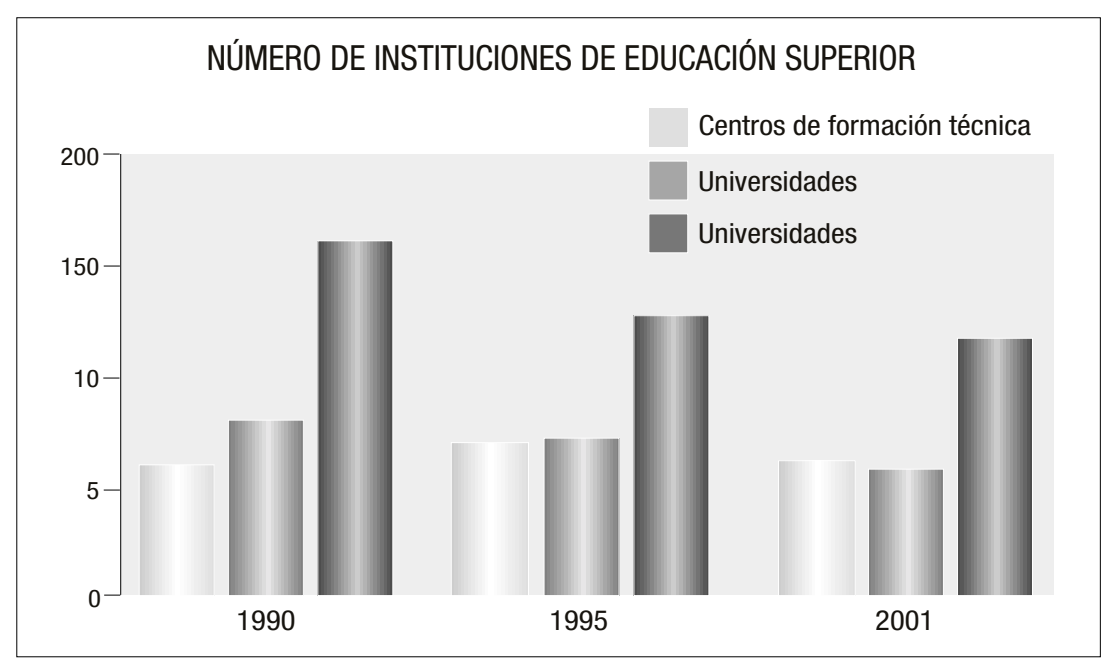




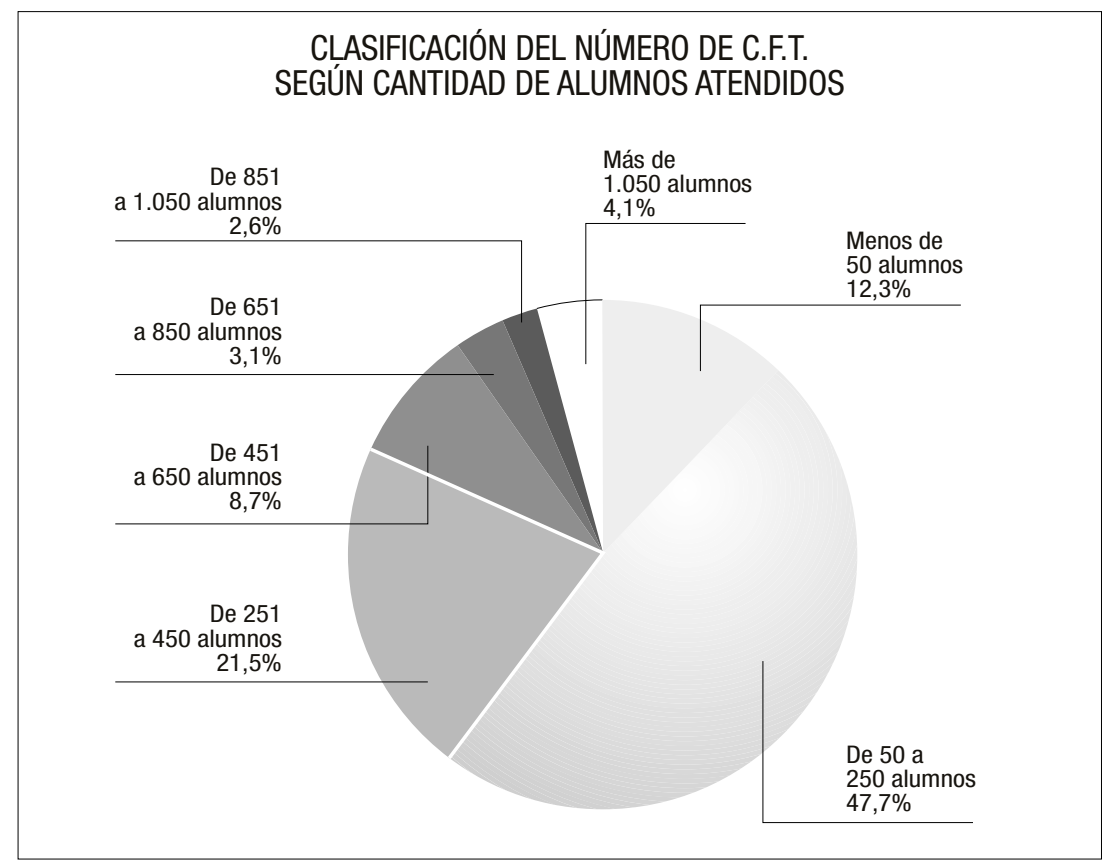

\section{FOMENTO A LA EQUIDAD}

Uno de los rasgos que caracteriza el desarrollo de la educación postsecundaria en general y en Chile en particular, es el crecimiento de la matrícula y su extensión a grupos tradicionalmente excluidos de ella. La población que llega hoy a la educación superior es mucho más diversa de lo que ha sido siempre: proviene de distintos sectores socioculturales y socioeconómicos, con una diversidad de intereses y características y, en muchos casos, ingresa después de un período de trabajo.

Esta población tiene necesidades diferentes a las tradicionales y, por consiguiente, la responsabilidad pública por asegurar la igualdad de oportunidades incluye aquí no sólo acciones destinadas a facilitar el acceso a diversas opciones de estudio, sino también a promover la permanencia de estos estudiantes en las carreras elegidas, así como su egreso a su debido tiempo. 
Asimismo, es un requisito de equidad impulsar acciones tendientes a mejorar la articulación entre los distintos niveles de estudio y la flexibilidad en las ofertas educativas, con el fin de promover la formación continua y permanente de la fuerza de trabajo, y establecer vínculos entre los programas de capacitación y la educación formal conducente a título. Lo anterior debe efectuarse en el marco de una preocupación constante por la calidad, cuyo logro es también una condición esencial de la equidad.

El concepto de educación permanente o para toda la vida podría ser el eje que permita articular las distintas fases de la educación secundaria y superior, posibilitando la superación de las divisiones entre educación formal y formación profesional o capacitación y también la noción de educación para el trabajo, que puede estrechar excesivamente los objetivos. En este sentido, es necesario considerar la incorporación del sistema modular en el diseño de las mallas curriculares, que intenta hacer convergente el propósito de proporcionar la formación para una adecuada inserción laboral con el de una efectiva educación continua.

Otra necesidad de equidad se relaciona con un desarrollo equilibrado de la oferta educativa de nivel técnico en las distintas regiones del país. Es necesario que los alumnos encuentren en sus regiones oportunidades de estudio interesantes y de calidad, vinculadas al empleo y a las necesidades de los diferentes ámbitos de la comunidad regional.

Finalmente, la equidad también exige la provisión de una adecuada, permanente y oportuna información pública destinada a los usuarios del sistema y a quienes contribuyen a su financiamiento, particularmente los estudiantes.

\section{INSTRUMENTOS Y LÍNEAS DE ACCIÓN}

Partiendo de la base de que la calidad y la equidad son los ejes orientadores de las políticas en el sector, se ha podido avanzar hacia la definición de los principales instrumentos de política: la regulación, el financiamiento y la información, que están en la base de las líneas de acción. 


\section{La regulación}

Se trata fundamentalmente del perfeccionamiento de los mecanismos de aseguramiento de la calidad y el logro de una mayor articulación entre la educación secundaria y la superior y las distintas instancias de la educación postsecundaria.

La experiencia adquirida durante la última década ha puesto en evidencia la necesidad de revisar y compatibilizar las estrategias utilizadas para asegurar la calidad de la formación de técnicos de nivel superior.

Considerando lo anterior, el Ministerio de Educación está realizando un profundo proceso de análisis y revisión de los mecanismos de supervisión y acreditación de los centros de formación técnica que son de su responsabilidad. Esto ha significado tomar en cuenta aspectos normativos, conceptuales, de procedimiento y de gestión del proceso, lo que se ha traducido en la revisión de los criterios de evaluación y los instrumentos y procedimientos de supervisión y acreditación ${ }^{2}$. Con esta finalidad, se han organizado grupos de trabajo conformados por profesionales de la División y otras personas relacionadas con estas materias provenientes del Consejo Superior de Educación, de la Comisión Nacional de Acreditación de Pregrado, de la Fundación Chile, de instituciones formadoras, del sector productivo, etcétera.

En esta misma línea, se ha reestructurado el Departamento de Centros de Formación Técnica del Ministerio, en cuyo diseño se ha establecido la creación de un Comité Asesor, que contempla la participación de diversos actores vinculados a este ámbito, incorporando también a la empresa. Su función es servir como órgano consultivo del Ministerio de Educación en las materias relacionadas con los sistemas de regulación de los centros de formación técnica.

Con el propósito de asegurar la calidad, principalmente a través del Fondo Competitivo para Técnicos del MECESUP, se ha incentivado

2. Esto incluye la elaboración de instrumentos facilitadores de este proceso, el desarrollo de mecanismos de gestión para un uso adecuado de los equipos de pares evaluadores que intervienen, la implementación de sistemas de información de seguimiento de la supervisión y acreditación, etcétera. 
la acreditación como requisito institucional para presentar los proyectos concursables. Asimismo, se está promoviendo la acreditación voluntaria de carreras de nivel técnico superior de instituciones autónomas, para lo cual la Comisión Nacional de Acreditación de Pregrado ha constituido un Comité Técnico integrado por especialistas, que ha definido criterios de evaluación para estas carreras.

Un aspecto fundamental es avanzar hacia la generación de esquemas que permitan una mayor articulación entre los distintos niveles de formación, para lo cual deben considerarse los modelos exitosos aplicados en otros países. Así, por ejemplo, cada vez se acepta más la presencia del currículum basado en competencias, que se considera un mecanismo facilitador de la articulación vertical, o el desarrollo de programas modulares.

En este sentido, el Fondo Competitivo para Técnicos del MECESUP está estimulando la presentación de proyectos con diseños curriculares flexibles y la conformación de redes entre instituciones, para optimizar el uso de recursos y facilitar la articulación entre programas, niveles e instituciones.

Para todo lo anterior se hace imprescindible una capacitación continua para los distintos actores del sistema, con el fin de ofrecer las herramientas adecuadas que permitan llevar a cabo un efectivo proceso de modernización de los esquemas educativos y de gestión. Así por ejemplo, a través del MECESUP se organizan seminarios y talleres, mayoritariamente en regiones, sobre desarrollos curriculares basados en competencias, la vinculación educación técnica superiorempresa, la modernización de la gestión institucional mediante la aplicación de las tecnologías de información, etcétera. Igualmente, a las instituciones que desarrollan proyectos se les financian consultorías con la participación de expertos de países industrializados, para ayudar a la construcción de capacidad de gestión.

Por último, es imperativo perfeccionar algunas normas que guardan relación con la formación de técnicos de nivel superior. En consecuencia, es necesario efectuar modificaciones específicas que contribuyan a facilitar su mejoramiento o establecer incentivos para potenciarla. En primera instancia se puede identificar temas como los siguientes: 
- Marco jurídico regulatorio de los mecanismos de aseguramiento de la calidad.

- Mecanismos de fusiones, absorciones y consorcios que permitan la consolidación de instituciones pequeñas.

- Mecanismos de validación de estudios y reconocimiento de competencias.

- Estudio de modificaciones a la Ley de Donaciones, ya que actualmente los centros de formación técnica están impedidos legalmente para recibir donaciones.

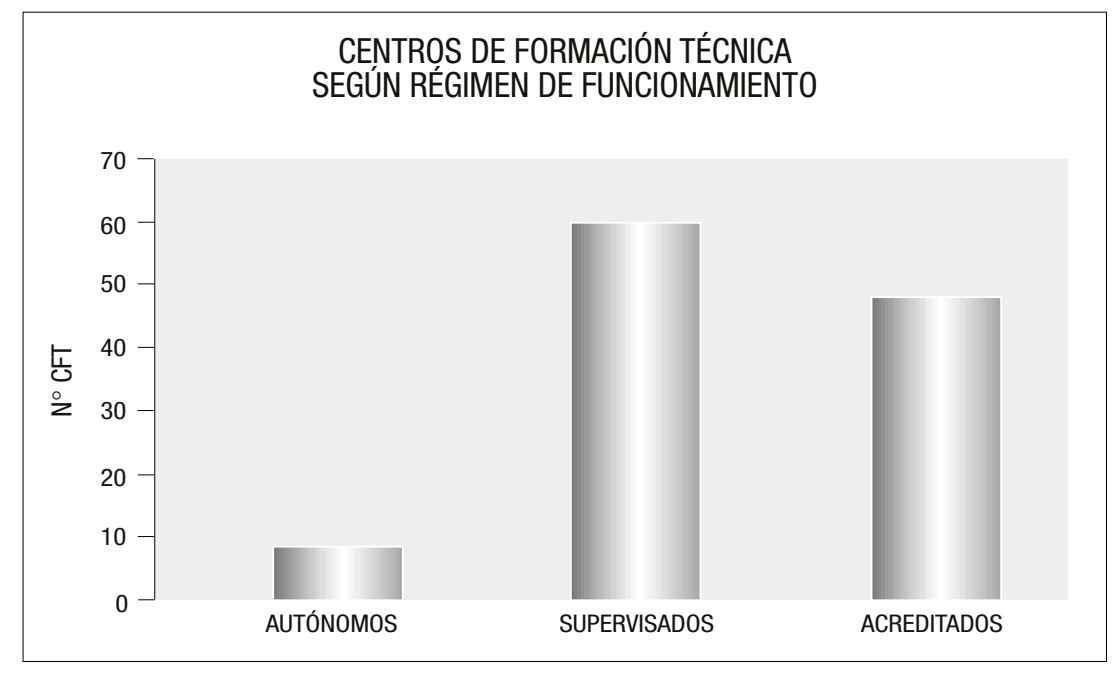

\section{El financiamiento}

Los esfuerzos se concentran en dos dimensiones principales: la definición de diversos mecanismos tendientes a mejorar las condiciones de ingreso a la educación superior de los estudiantes y la creación de programas que permitan obtener financiamiento para optimizar las instalaciones y los procesos formativos de las instituciones que ofrecen carreras técnicas.

Con respecto a lo primero, se ha impulsado el establecimiento de becas asignadas con criterios de calidad y equidad, y el acceso al crédito. 
Sin embargo, en este punto es muy importante recalcar que las instituciones donde los beneficiados realizan sus estudios deben someterse a procesos de evaluación y selección para estimular la calidad de la oferta educativa.

Una de las iniciativas más emblemáticas es el Programa de Becas Nuevo Milenio iniciado en el año 2001, dirigido a apoyar a estudiantes que siguen carreras técnicas de nivel superior en instituciones determinadas previamente. Por su intermedio ya se han otorgado 9.000 becas equivalentes a 2.700 millones de pesos. La gran mayoría de los beneficiados estudia en regiones y en centros de formación técnica.

En cuanto a lo segundo, en el Fondo Competitivo para Técnicos del MECESUP -que permite acceder a recursos para realizar proyectos que mejoren la calidad, pertinencia y articulación de la educación técnica de nivel superior-en los años 2000 y 2001 se han distribuido 7.200 millones de pesos y, para el 2002, se dispone de 4.000 millones. $^{3}$

Otra acción importante es la ampliación de la franquicia tributaria mediante la nueva Ley SENCE, que permite que los centros de formación técnica financien módulos orientados a entregar títulos de técnico superior basados en competencias laborales previamente autorizados por el Ministerio de Educación.

Las consecuencias de lo anterior se inscriben en la doble orientación ya mencionada: por un lado, se facilitará el acceso de trabajadores a la formación técnica superior, permitiendo financiamiento por medio de la franquicia, lo que hace prever un aumento de la cobertura en el sector. Por otro, se obligará a que los centros transformen su oferta curricular a través de la modularización, sobre la base de enfoques basados en competencias laborales.

Esto es significativo porque actualmente los cursos de capacitación son autosuficientes o terminales, no siguen necesariamente una línea formativa ni admiten certificaciones o equivalencias con el sistema educativo formal. Además, permite el

3. En los concursos 2000-2001 se han adjudicado 34 proyectos correspondientes a 23 instituciones. 
financiamiento de módulos de formación que en conjunto constituirán una carrera conducente a título y apunta con mayor precisión a la inserción laboral del educando una vez completado su ciclo de formación. Simultáneamente, este mecanismo facilita la articulación entre distintos niveles formativos y el desarrollo de un sistema de articulación vertical, aumentando la eficiencia global en la utilización de los recursos, facilitando la continuación de estudios y el acceso a los siguientes niveles mediante mecanismos de reconocimiento de competencias o conocimientos adquiridos.

En relación al enfoque de competencias laborales, hay que destacar que entre sus ventajas está que posibilita identificar los conocimientos, habilidades y actitudes que el mundo productivo requiere de la fuerza laboral; mejora la oferta de formación y capacitación, alineándola con esos requerimientos; reconoce las competencias de los trabajadores, independientemente de cómo las adquirieron y aumenta la competitividad empresarial a través de las personas.

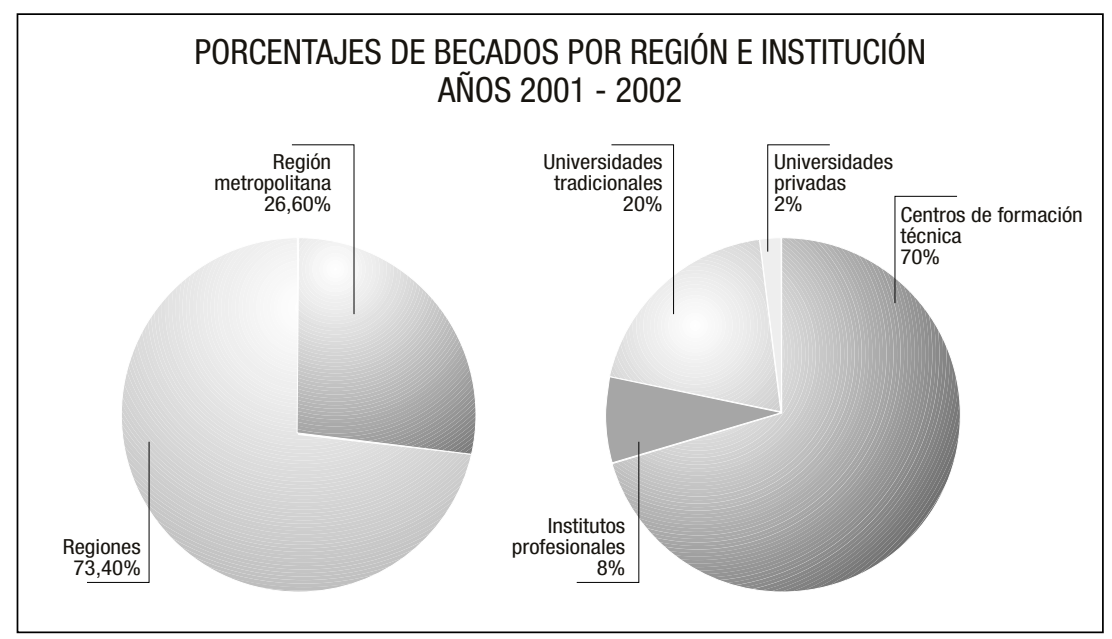



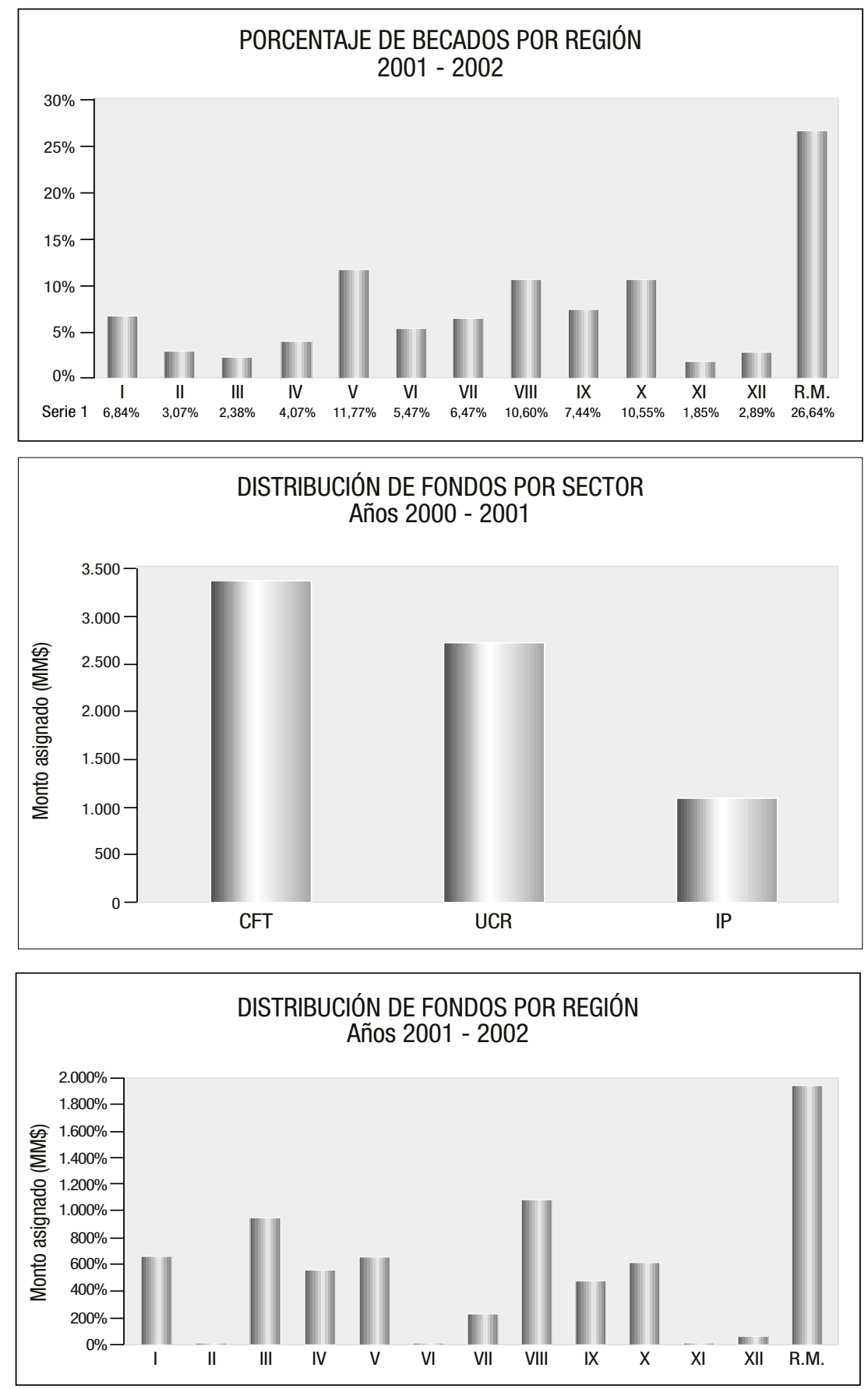


\section{La información}

El perfeccionamiento de los sistemas de información es un requisito tanto para la equidad como para la calidad. Se trata, entonces, de incorporar la oferta de estudios técnicos en los mecanismos de difusión que se establezcan en el campo de la información pública. Esto incluye proporcionar al máximo de usuarios antecedentes acerca de los programas que se ofrecen, abarcando indicadores de calidad y de oportunidades de empleo.

La página web www.mineduc.cl cumple estas funciones, ya que en ella se publica un Directorio que contiene información oficial de datos institucionales, carreras, sedes y estadísticas, matrícula, titulados y aranceles de las distintas carreras, así como glosario de términos.

Del mismo modo, la edición anual del Informativo Académico correspondiente a los centros de formación técnica, integra datos de carreras, matrícula, rendimiento académico, titulados, vacantes, arancel, cantidad de docentes, número de títulos de biblioteca, exigencias de ingresos, beneficios estudiantiles, etcétera.

Se está avanzando en un nuevo sistema nacional de información sobre educación superior que busca determinar un conjunto de indicadores relevantes para la toma de decisiones, cuyo fin es superar las asimetrías de información que existen actualmente, garantizando confiabilidad y validez. Para la implementación de este sistema es fundamental generar las condiciones que promuevan y contribuyan a desarrollar la capacidad de las instituciones para alimentarlo.

En síntesis, tanto las políticas como los instrumentos y líneas de acción que se han reseñado muestran un todo coherente y se reflejan en las diversas iniciativas destinadas a fortalecer la educación técnica superior, de manera de lograr una adecuada inserción laboral y social de importantes sectores de la población y, al mismo tiempo, aumentar la competitividad nacional en la producción de bienes y servicios. 